\title{
Publicaciones seriadas de la terapia ocupacional en latinoamerica ${ }^{1}$
}

\author{
Aleida Fernández Moreno \\ Terapeuta Ocupacional, Magistra en Desarrollo Educativo y Social, Doctora en Ciencias Sociales, \\ Niñez y Juventud, Coordinadora Académica de la Maestría en Discapacidad e Inclusión Social, \\ Profesora asociada de la Universidad Nacional de Colombia, Bogotá, Colombia
}

\begin{abstract}
Resumen: Este artículo presenta el panorama bibliográfico de las revistas de Terapia Ocupacional en cuatro países: Argentina, Chile, Colombia y Venezuela. Desde los inicios de la Terapia Ocupacional en los países de la región latinoamericana, fueron creados distintos programas de formación a nivel técnico, posteriormente, a mediados de los ochenta, la mayoría de programas se reconfiguraron a nivel profesional, al incorporar trabajos investigativos como requisito de grado. En los primeros años el soporte bibliográfico se importaba de construía con base a contextos angloparlantes. La mayor parte del revistas específico latinoamericana da área comenzó a finales de los noventa. Su desarrollo es desigual en el tiempo, el mantenimiento de una mayor tradición de la publicación de Libros. El presente trabajo discute discute los inicios, el desarrollo, la decadencia y el resurgimiento de las publicaciones seriadas en el contexto latinoamericano. Resulta que todos los esfuerzos se requieren para reactivar la publicación en revistas propias de terapia ocupacional y usar los recursos digitales para lograrlo, son pasos hacia la apropiación social del conocimiento y la consolidación de la Terapia Ocupacional como profesión.

Palabras clave: Publicaciones Periódicas, Documentos en Texto Completo, Resumen e Indización, Terapia Ocupacional.
\end{abstract}

\section{Publicaçóes periódicas da terapia ocupacional na América Latina}

Resumo: Este artigo apresenta o panorama bibliográfico de revistas de Terapia Ocupacional de quatro países: Argentina, Chile, Colômbia e Venezuela. Desde o início da Terapia Ocupacional nos países da região latino-americana, foram criados vários programas de formação em nível técnico. Posteriormente, em meados dos anos oitenta, a maioria dos cursos se reconfigurou no nível profissional, ao incorporar trabalhos de pesquisa como requisito para obtenção do diploma. Nos primeiros anos, o apoio bibliográfico se construiu com base na produção anglo-saxônica. A maior parte das revistas específicas latino-americanas da área começou ao final dos anos noventa. Seu desenvolvimento é desigual no tempo, mantendo uma maior tradição de publicação em livros. O presente trabalho discute o início, o desenvolvimento, a queda e ressurgimento das publicações periódicas em Terapia Ocupacional no contexto latino-americano. Conclui-se que são necessários todos os esforços para reativar a publicação em revistas próprias da área de Terapia Ocupacional e usar os recursos digitais para desenvolvê-la, visando à apropriação social do conhecimento e à consolidação da Terapia Ocupacional como profissão.

Palavras-chave: Publicações Periódicas, Documentos com Texto Completo, Resumos e Indexação, Terapia Ocupacional. 


\title{
Serial publications in occupational therapy in Latin America
}

\begin{abstract}
This article presents a bibliographic overview on Occupational Therapy journals in four countries: Argentina, Chile, Colombia and Venezuela. Since the beginning of occupational therapy in Latin American countries, several training programs at the technical level were created. In the mid 1980s, most courses were reconfigured at the professional level, incorporating researches as a requirement for obtaining the degree. In the early years, the literature was built on the supporting basis of the North American production. Most of the specific Latin American journals in the area began in the late nineties. It has developed unevenly through time, maintaining the greater tradition of publishing books. This paper discusses the beginning, development, decline and resurgence of Occupational Therapy periodicals in Latin American. It was possible to conclude that all efforts are needed to revive the publication of journals specific to the area of occupational therapy and use digital resources to develop them, aiming at the social appropriation of knowledge and consolidation of occupational therapy as a profession.
\end{abstract}

Keywords: Periodicals as Topic, Full-Text Documents, Abstracting and Indexing, Occupational Therapy.

\section{Introducción}

Desde los inicios de la Terapia Ocupacional en los países de la región latinoamericana, fueron creados distintos programas de formación a nivel técnico: en Argentina, Brasil, México y Venezuela a finales de la década de los cincuenta, en Chile y Colombia en la década de los sesenta en, y en los setenta en Perú; posteriormente, a mediados de los ochenta, la mayoría de programas se reconfiguraron a nivel profesional, al incorporar trabajos investigativos como requisito de grado.

En los primeros años el soporte bibliográfico se importaba de construía con base a contextos angloparlantes: las versiones de la terapéutica de Mac Donald, así como la Terapéutica Ocupacional Willard and Spackman fueron unos de los libros más conocidos en Latinoamérica. Estos textos han sido traducidos al español y al portugués durante décadas. Estos textos fueron usados para definir la terapia ocupacional e identificar las áreas de intervención, así como los procedimientos de rehabilitación que se requerían según la patología atendida.

Sin embargo, una constante en Latinoamérica es que la amplia divulgación del conocimiento profesional y científico no se deriva estrictamente de los libros, sino de la publicación en revistas periódicas.

Tejada (2012), señala que desde la creación de las revistas científicas (en 1665 se publicó la primera revista Le Journal des Sçavans), se han publicado miles de artículos de investigación, revisión, informes, discusiones, cartas al editor, difusión de eventos, entre otros, que han permitido los grandes avances de las disciplinas y las ciencias; desde tiempos antiguos es evidente que cualquier editor conoce la importancia de velar por la calidad editorial de sus contenidos. En el contexto de la Terapia Ocupacional, fueron las Asociaciones de carácter científico las que patrocinaron las revistas nacionales y aseguraron su calidad. Así, los avances regionales se fueron registrando con la iniciación y progresiva

Tabla 1. Revistas publicadas en Argentina, Chile, Colombia y Venezuela.

\begin{tabular}{|c|c|c|c|}
\hline Revista (Publicación) & Año (Período) & Institución & ISSN \\
\hline $\begin{array}{l}\text { Boletines de la Asociación Argentina de } \\
\text { Terapia Ocupacional }\end{array}$ & $\begin{array}{c}1970 \ldots \\
\text { Cuatrimestral }\end{array}$ & $\begin{array}{c}\text { Asociación Argentina de Terapia } \\
\text { Ocupacional (AATO). }\end{array}$ & No \\
\hline Materia Prima & $\begin{array}{l}\text { 1996-2000 } \\
\text { Bimensual }\end{array}$ & & Sí \\
\hline El Puente & $1998-2002$ & $\begin{array}{c}\text { Universidad Nacional de Mar } \\
\text { del Plata }\end{array}$ & No \\
\hline Cuadernos de Terapia Ocupacional & $1996 \ldots$ & $\begin{array}{l}\text { Universidad Nacional de } \\
\text { Quilmes }\end{array}$ & \\
\hline $\begin{array}{l}\text { Revista Argentina de Terapia Ocupacional } \\
\text { http://www.ocupate.vaneduc.edu.ar }\end{array}$ & & $\begin{array}{l}\text { Universidad Abierta } \\
\text { Interamericana }\end{array}$ & \\
\hline
\end{tabular}

Fuente: Elaboración propia (2011), compilando la información reportada por la T.Os Sara Daneri y Victoria Toscano. 
Tabla 1. Continuación...

\begin{tabular}{|c|c|c|c|}
\hline Revista (Publicación) & Año (Período) & Institución & ISSN \\
\hline $\begin{array}{l}\text { Revista Chilena de Terapia de Terapia } \\
\text { Ocupacional } \\
\text { http://www.revistaterapiaocupacional.cl }\end{array}$ & 2001 & Universidad de Chile & Sí \\
\hline \multicolumn{4}{|c|}{$\begin{array}{l}\text { Fuente: Elaboración propia (2011), compilando la información reportada por la T.O. Dalila Goudeau de la } \\
\text { Universidad de Playa Ancha, Chile. }\end{array}$} \\
\hline Revista (Publicación) & Año (Período) & Institución & ISSN \\
\hline Revista Acción & & $\begin{array}{l}\text { Asociación Colombiana de Terapia } \\
\text { Ocupacional (ACTO) }\end{array}$ & No \\
\hline Revista de la Ocupación Humana & $\begin{array}{l}1997-2007 \\
\text { Semestral }\end{array}$ & $\begin{array}{l}\text { Asociación Colombiana de Terapia } \\
\text { Ocupacional (ACTO) }\end{array}$ & Sí \\
\hline Revista de la Ocupación Humana & $2012 \ldots$ & $\begin{array}{l}\text { Colegio Colombiano de Terapia } \\
\text { Ocupacional }\end{array}$ & \\
\hline Ukupari & $\begin{array}{l}2010 \\
\text { Primer ejemplar }\end{array}$ & $\begin{array}{c}\text { Colectivo de estudiantes } \\
\text { de la Universidad } \\
\text { Nacional de Colombia }\end{array}$ & \\
\hline \multicolumn{4}{|c|}{$\begin{array}{l}\text { Fuente: Elaboración propia (2011), compilando la información reportada por la T.O. Aida Navas de Serrato, } \\
\text { Presidenta del Colegio Colombiano de Terapia Ocupacional. }\end{array}$} \\
\hline Revista (Publicación) & Año (Período) & Institución & ISSN \\
\hline $\begin{array}{l}\text { Revista electrónica de Terapia } \\
\text { Ocupacional Venezolano. Espacio } \\
\text { T.O. Venezuela } \\
\text { http://www.espaciotovenezuela.com }\end{array}$ & $\begin{array}{l}2008 \\
\text { (Bimensual) }\end{array}$ & $\begin{array}{l}\text { Federación Venezolana de } \\
\text { Terapeutas Ocupacionales }\end{array}$ & Sí \\
\hline
\end{tabular}

Fuente: Elaboración propia (2011) a partir de los aportes de María Antonieta Rivas de Puche y Anggeli González.

publicación de las primeras revistas. A continuación en la Tabla 1 se reporta el desarrollo de las revistas profesionales en cuatro (4) países: Argentina, Chile, Colombia y Venezuela ${ }^{2}$.

En Argentina a finales del siglo XX, se alcanzó un número sin precedente de publicaciones en la región de más de cuatro revistas (4) de alto nivel, destacándose en su producción las patrocinadas por la Asociación Argentina de Terapia Ocupacional (AATO), y por las Universidades Nacionales de Mar del Plata y de Quilmes. Las primeras publicaciones de la AATO fueron boletines que aparecen con el Volumen 1 Año 1 en Abril de 1970, publicación cuatrimestral de aproximadamente 20 páginas por número que contaba con ISSN ${ }^{3}$. Ya en 1996, aparece Materia Prima, revista de carácter bimensual con ISSN (0328-7890), que se publica hasta junio del 2000. En Mar del Plata desde 1998 hasta diciembre del 2000, se publicó El Puente, con artículos y reseñas relacionadas con la cátedra práctica clínica de la salud y la seguridad social, se trataba de publicación coordinada desde la Universidad Nacional del Mar del Plata. También en diciembre de 1996 se registran los denominados Cuadernos de Terapia Ocupacional, publicación a cargo de Universidad Nacional de Quilmes. Hay reporte de una publicación de un colectivo de estudiantes, e infortunadamente no se logró acceder a dicha información.

Sin embargo, la mayoría de las revistas, entre ellas Materia Prima, El Puente, Cuadernos de Terapia Ocupacional, dejaron de ser publicadas por falta de financiación, en tiempos del "corralito de piedra" no pudieron continuar su importante labor y tampoco lograron llegar al siglo XXI.

En Chile, en los albores del siglo XXI, a partir del 2001 se publica la Revista Chilena de Terapia Ocupacional, a cargo de la Escuela de Terapia Ocupacional de la Facultad de Medicina de la Universidad de Chile. En la actualidad esta revista tiene en la virtualidad un aliado, dado que se trata de una publicación que vía Internet está siendo consultada por colegas de toda Latinoamérica.

En Colombia fue la Asociación Colombiana de Terapia Ocupacional (ACTO), que como asociación de carácter científico se encargó de publicar la revista de la Ocupación Humana (1997-2007). Esta institución cumplió un importante papel en la difusión del conocimiento. Así mismo, en el 2010, se registra una iniciativa de los estudiantes de la Universidad Nacional de Colombia, donde se proyectan nuevos números de la revista Ukupari. 
En la actualidad la antigua Asociación Colombiana de Terapia Ocupacional (ACTO), es transformada por las exigencias de la Ley colombiana en Colegio Colombiano de Terapia Ocupacional (CCTO) y se proyecta para este ańo 2012 el relanzamiento de la Revista de la Ocupación Humana, patrimonio de los terapeutas ocupacionales de este país.

En Venezuela, la nueva revista venezolana de terapia ocupacional, Espacio T.O, circula vía Internet de carácter gratuito, según la información recolectada con el respaldo de la Federación Venezolana de Terapeutas Ocupacionales.

\section{Resultados}

El inicio de formación a nivel técnico en latinoamérica fue una de las razones por la que los programas fueron subsidiarios de los avances teóricos y conceptuales registrados en los países angloparlantes, especialmente de Inglaterra y Estados Unidos. Desde la década de los ochenta y con el fortalecimiento de las Asociaciones profesionales y Sociedades científicas, se empezó a prestar más atención a las publicaciones seriadas, algunos boletines se convierten en revistas y se nutren de los trabajos de los profesionales y de estudiantes de cada país. A finales de los noventa la mayoría de las revistas de los países de la región están en pleno auge, sin embargo, al iniciar el siglo XXI, los problemas de financiación agobian la producción de las publicaciones y algunas de ellas salen de circulación.

La desaparición de las revistas impresas, como en el caso de Argentina y Colombia, es una muestra de las dificultades para mantener las publicaciones periódicas de este tipo en Latinoamérica, y en particular, si es en torno a la Terapia Ocupacional. Razones de orden financiero se imponen, pero también los criterios de visibilidad de su producción, a los cuales deben atender los Terapeutas académicos, y especialmente los investigadores que buscan la indización de sus textos en revistas reconocidas, de amplia circulación y en buena medida están prefiriendo publicar en el extranjero y no en sus países de origen, tendencia que se ve también en otras áreas del conocimiento. De lo anterior se desprenden efectos negativos, dado que la dispersión de la producción académica en revistas extranjeras, así como en otras áreas, hace que se pierda el capital del conocimiento logrado.

La publimetría ${ }^{4}$ actual está referida a altos estándares de circulación y consulta, lo cual hace que los profesionales de gran trayectoria busquen publicar en las revistas catalogadas de alto nivel de impacto, es decir, la mayoría busca publicar en revistas extranjeras y en lo posible norteamericanas de alto ranking. Además de enviar sus ensayos, artículos investigativos y reseńas, entre otros, serán publicadas en revistas de otras profesiones que, si bien visibilizan la Terapia Ocupacional en otros contextos, hacen muy difícil catalogar y acceder a la producción bibliográfica de los colegas y de las temáticas en torno al campo. Los autores citantes Solana y Liberatore (2008), han distinguido a tres grupos: autores citantes nacionales, es decir, que pertenecen al mismo país que edita la revista; autores citantes regionales, es decir de la región iberoamericana; y autores citantes internacionales. Siguiendo este parámetro, la visibilidad internacional de autores terapeutas es baja y aún no existen indicadores de autores citantes contabilizados (es el portal en español el que más alto índice de consulta tiene; en este espacio virtual se reseñan las revistas latinoamericanas).

En el caso de Colombia, Tejada (2012), retoma Publindex- sistema de Colciencias, y muestra como la medición de grupos de investigadores cambiará; así el Publindex se convierte en una exigencia nacional para renovar las dinámicas de los incentivos del Sistema nacional de ciencia y tecnología (SNCyT), y por la búsqueda de la "internacionalización" académica. La base serán los indicadores de medición y evaluación de la ciencia y tecnología - cienciometría y bibliometría -, y tendremos que aprender a conocer sobre las citaciones, el factor de impacto, los cuartiles, índices de citaciones, entre otros.

\section{Prospectiva}

La globalización e internacionalización de la actualidad implica para las revistas una condiciones que no favorecen su desarrollo a nivel nacional y regional. La mayoría de las revistas latinoamericanas no están incluidas en el Web of Science o Scopus ${ }^{5}$, son pocas las que hacen parte de Lilacs (sistema que hace más de 25 años es el más importante y abarcador índice de la literatura científica y técnica en Salud de América Latina y de Caribe).

Hacer uso de los "repositorios" de las Universidades, que en su mayoría hacen uso del principio del copy-left, dejan disponible para el público en general las investigaciones que los estudiantes de pregrado de Terapia Ocupacional realizan como requisito de grado; estas se convierten en un tesoro documental, actualizado y de impacto. Así, el uso de las Tecnologías de la información y la comunicación (TIC), son fundamentales para divulgar las investigaciones, la sistematización de 
experiencias y otra producción bibliográfica en Terapia Ocupacional. Como ejemplo, se destaca la iniciativa de la Universidad Nacional de Colombia en este sentido, ya que pueden consultarse las publicaciones gratuitas en la URL http://www. bdigital.unal.edu.co.

Según Tejada, para el presente siglo, el énfasis ha de ser Digital, pues desde la llegada de Internet la migración de las RCC a página web se ha incrementado; inició en la Web 1.0 y ha evolucionado a la Web 2.0, o la Ciencia 2.0. Igualmente los e-book, tabletas y celulares inteligentes serán el futuro que conlleve a tener los artículos de las revistas en los e-readers, PDF, epub y mobi, generando enlaces hipertextuales, nuevos formatos de audio y vídeo, etc. Todos estos fenómenos exigen que la información se encuentre más organizada, normalizada, con los metadatos que permitan realizar los análisis de información, medición y visualización. Estás dinámicas proporcionan a las revistas canales más agiles y eficaces de comunicación con los lectores, los autores y los investigadores. Propiciar el incremento de publicaciones virtuales, especialmente de las revistas de Terapia Ocupacional de Argentina, Chile y Venezuela, además de los altos índices de consulta que reporta la revista virtual de terapia ocupacional española (con más de 60.000 visitas mensuales al portal desde el año 2000), invitan a otras publicaciones a identificar si esta es la vía a seguir para la difusión del conocimiento, así como a establecer y definir nuevos rumbos para el desarrollo del campo y la producción de sus debates y nuevos conocimientos.

Dado que los medios de publicación y difusión de información se están modificando tanto con lo virtual, textos interactivos en temas básicos podrían ser abordados por los estudiantes, obteniendo así niveles más altos de aprendizaje. Las guías de intervención y de práctica basadas en la evidencia podrían ser socializadas y consensuadas por expertos latinoamericanos como una potente forma de transformar las formas de construir conocimiento propio de la Terapia Ocupacional.
Por otra parte, según el Catálogo de publicaciones de la Confederación Latinoamericana de Terapia Ocupacional (CONFEDERACIÓN..., 2009, 2011), se registra en toda la región un incremento en la publicación de libros, capítulos, cartillas y otros textos relacionados. Estos esfuerzos son relevantes, por lo que debemos buscar nuevas formas para poder compartirlos, quizás apoyándonos en las nuevas tecnologías, creando repositorios y portales donde podamos subir los textos y manejar un copy-left que haga accesible el conocimiento de nuestra profesión.

Se trata de incrementar la circulación de información contenida en el Catálogo de publicaciones de la CLATO, las Asociaciones, Sociedades Científicas, los Colegios Profesionales, las federaciones y todas aquellas instituciones que manejen información prioritaria para la Terapia Ocupacional, especialmente en lo que a publicaciones concierne.

Todos los esfuerzos para reactivar la publicación en revistas propias de terapia ocupacional y usar los recursos digitales para lograrlo, son pasos hacia la apropiación social del conocimiento y la consolidación de la Terapia Ocupacional como profesión.

\section{Referencias}

CONFEDERACIÓN LATINOAMERICANA DE TERAPIA OCUPACIONAL - CLATO. Catálogo de publicaciones de la Confederación Latinoamericana de Terapia Ocupacional. Santiago: CLATO, 2009.

CONFEDERACIÓN LATINOAMERICANA DE TERAPIA OCUPACIONAL - CLATO. Catálogo de publicaciones de la Confederación Latinoamericana de Terapia Ocupacional. Santiago: CLATO, 2011.

SOLANA, V.; LIBERATORE, G. Visibilidad internacional de las revistas iberoamericanas de Bibliotecología y Documentación International visibility of the IberoAmerican journals of library and informationscience. Revista Española de Documentación Cientifica, Madrid, v. 31, n. 2, p. 230-239, abr./jun. 2008. Disponible en: <redc. revistas.csic.es/index.php/redc/article/download/426/438>. Consultado el: 10 jun. 2012.

TEJADA, M. A. Entre tendencias o disidencias. El futuro de las Revistas Científicas Colombianas. Unilibros de Colombia, Bogotá, n. 19, p. 108-111, 2012. 


\section{Notas}

${ }^{1}$ Texto basado en la Ponencia Panorama Bibliográfico de la Terapia Ocupacioanl en Latinoaméca, presentada en el IX Congreso Latinoamericano de Terapia Ocupacional. Sao Pablo (2011).

${ }^{2}$ Brasil es el país que reporta a nivel de la profesión una productividad comparativamente alta en términos de publicaciones existentes en este importante país de la región, fue la T.O. Fátima Corrêa en Octubre de 2011, quien dio cuenta de esta producción en el XXV Congreso Latinoamericano de Terapia Ocupacional llevado a cabo en Sao Pablo de las publicaciones y es a ella a quien se debe consultar en este campo.

${ }^{3}$ El ISSN (Número Internacional Normalizado de Publicaciones Seriadas) es básicamente un código mediante el cual una publicación periódica será reconocida internacionalmente. Nació en 1973 por acuerdo entre el gobierno francés y la Organización de las Naciones Unidas para la Educación (UNESCO), estas hicieron una organización con todos sus contactos y la comunidad de librerías, pues era necesario lograr el ISSN para las revistas, artículos técnicos y científicos.

${ }^{4}$ Publindex en Colombia.

${ }^{5}$ El Web of Science (WoS) es un índice de citas y el Scopus se usa para de búsquedas avanzadas de autores como el Author Search en Scopus.

${ }^{6}$ Universidad Nacional de Colombia. Disponible en: <http://www.bdigital.unal.edu.co>. 